\title{
Unilateral substitutability implies substitutable completability in many-to-one matching with contracts *
}

\author{
Sangram Vilasrao Kadam ${ }^{\dagger}$
}

April 6, 2015

\begin{abstract}
We prove that the unilateral substitutability property introduced in Hatfield and Kojima [2010] implies the substitutable completability property from Hatfield and Kominers [2014]. This paper provides a novel linkage between these two sufficient conditions for the existence of a stable matching in many-to-one matching markets with contracts. A substitutable completion of a preference is a substitutable preference created by adding some sets of contracts to the original preference order. We provide an algorithm which when operated on the unilaterally substitutable preferences produces such a substitutable completion. Thus it provides a constructive proof of the connection between the two properties.
\end{abstract}

${ }^{*}$ I am deeply indebted to Scott Kominers and Eric Maskin for extremely helpful discussions and detailed comments on this paper. I would also like to thank Mira Frick, Drew Fudenberg, Ben Golub, Yuhta Ishii, Divya Kirti, Fuhito Kojima, Maciej Kotowski, Assaf Romm, Al Roth, Ran Shorrer, Tayfun Sönmez, Neil Thakral for helpful discussions and comments and the two anonymous referees for valuable feedback which improved the exposition and the relevance of the results. All the remaining errors are mine.

${ }^{\dagger}$ Department of Economics, Harvard University email: svkadam@fas.harvard.edu. 


\section{Introduction}

The literature on many-to-one matching markets with contracts started with the seminal contributions by Kelso and Crawford [1982 $]^{1}$ and Hatfield and Milgrom [2005].2 ${ }^{3}$ The practical applications of these markets with contracts have recently been investigated in some interesting contexts like cadet branching (Sönmez [2013], Sönmez and Switzer [2013]), matching with regional caps (Kamada and Kojima [2012, 2015a]), and diversity design in school choice (Kominers and Sönmez [2013]).

Roth [1990] described the importance of stability for practical matching markets. He observed that the markets which generated a stable outcome continued to operate over longer periods of time than the ones which did not guarantee this property. For many-to-one matching with contracts, the literature has provided many conditions on the agents' preferences which are sufficient for stability, e.g. substitutability (Kelso and Crawford [1982], Roth [1984]), unilateral substitutability (Hatfield and Kojima [2010]), bilateral substitutability (Hatfield and Kojima [2010]), and substitutable completability (Hatfield and Kominers [2014]). However, the literature has not fully explored the connections between these sufficient conditions, which might be useful for practical applications. This paper shows that unilateral substitutability implies substitutable completability.

The preference of an agent on the many-side of the market satisfies the substitutability condition when the agent does not have any complementarities between contracts. ${ }^{4}$ In other words, the agent views each contract independently and never finds a contract that is rejected from some set of contracts to be acceptable only in the presence of another contract. A many-to-one preference of an agent satisfies the substitutable completability condition if there is a substitutable completion, i.e. a certain 'related' substitutable preference in the many-to-many setting for that agent. The preference of an agent has the unilateral substitutability property when the preference exhibits complementarities, if any, of only a certain kind; put differently, there may be certain 'permissible' violations of the substitutability condition.

Allowing for a broader class of complementarities gives the bilateral substitutability condition, and further expanding the 'allowed' set of violations of the substi-

\footnotetext{
${ }^{1}$ Kelso and Crawford [1982] builds on the analysis of Crawford and Knoer [1981].

${ }^{2}$ This generalized the many-to-one matching market from Gale and Shapley [1962]. That has in turn been further extended and generalized to interesting domains, viz. supply chain networks (Ostrovsky [2008], Hatfield and Kominers [2012]) and many-to-many matching markets with contracts (Hatfield and Kominers [2013]).

${ }^{3}$ Echenique [2012] has shown the surprising isomorphism between Kelso and Crawford [1982] and Hatfield and Milgrom [2005].

${ }^{4}$ All the relevant conditions are defined in section 2.2.
} 
tutability condition yields weak substitutability condition (abbreviated as W.Sub.). The weak substitutability condition is the strongest known necessary condition ${ }^{5}$ for stability. The following description and the two Venn diagrams summarize the relationships between these conditions that are known from the existing literature.

$$
\begin{aligned}
\text { substitutability [Sub.] } & \Rightarrow \text { unilateral substitutability [U.Sub.] } \\
& \Rightarrow \text { bilateral substitutability [B.Sub.] } \\
\text { substitutability } & \Rightarrow \text { substitutable completability [Sub.Comp.] } \\
\text { bilateral substitutability } & \nRightarrow \text { substitutable completability } \\
\text { substitutable completability } & \nRightarrow \text { bilateral substitutability }
\end{aligned}
$$
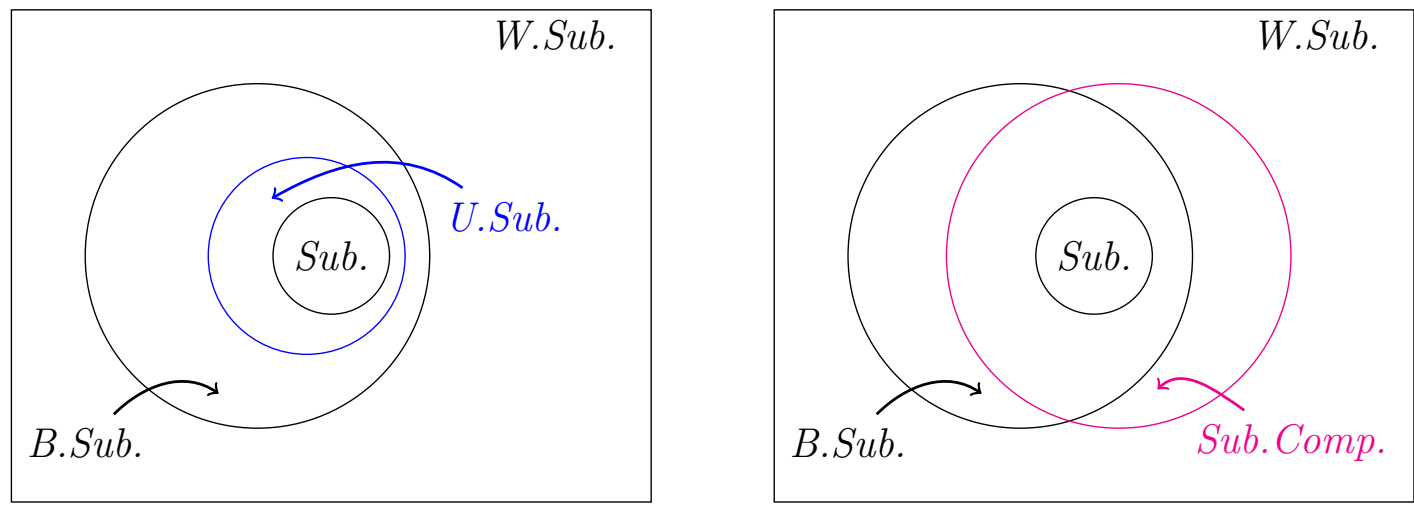

By proving that unilateral substitutability implies substitutable completability, we are able to provide the following unified Venn Diagram.

\footnotetext{
${ }^{5}$ The necessary condition implies that if there is an agent with a preference not satisfying the weak substitutability condition then there exists a setup of weak substitutable preferences for other agents such that no stable matching exists as proved in Hatfield and Kojima [2008].
} 


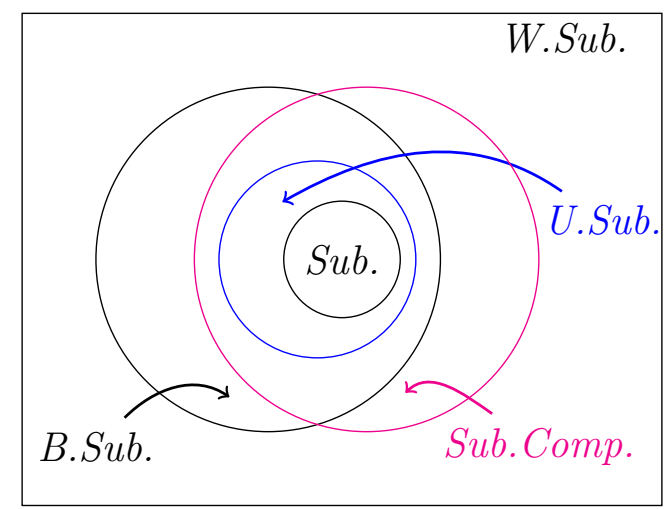

To fix ideas, consider the many-to-one matching setting of hospitals and doctors where a hospital can sign at most one contract with each of (possibly) many doctors. Each doctor can sign at most one contract. A set of contracts which involves multiple contracts with at least one doctor is termed an infeasible set. A substitutable completion of a preference, more precisely, is defined as a substitutable preference, i.e. a preference without any violations of the substitutability condition, created by the addition of infeasible sets to the original preference order. A preference that has a substitutable completion is defined as substitutably completable. Hatfield and Kominers [2014] give this definition involving the addition of infeasible sets to preference orders and provide techniques that work for some class of preferences, namely, slot-specific preferences and task-specific preferences.

In our proof, we provide an algorithm which for any unilaterally substitutable preference arrives at a substitutable completion. The algorithm ensures that the additions of infeasible sets, as allowed by the definition of completion, are at the 'right' places in the preference order, ensuring that the existing violations of the substitutability property are sequentially eliminated and that no new violations are created. Thus with the algorithm, we provide a constructive proof that unilateral substitutability implies substitutable completability.

The unilateral substitutability property was found to be relevant in the case of the cadet branching market (Sönmez and Switzer [2013]). It guaranteed the existence of a stable matching in that setting although the strongest of the sufficient conditions above, i.e. substitutability, was not satisfied. The substitutable completability property gives the intuitive understanding that in the setting of many-to-one matching market with contracts, there exists a stable matching which is a projection from the many-to-many matching market with contracts setting to the many-to-one case.

Moreover, in the case of matching with regional caps, Kamada and Kojima [2015a] (essentially) use the substitutable completability property while connecting their 
setting to the matching with contracts framework. ${ }^{6}$ They view regions and doctors as the two sides of the matching market instead of hospitals and doctors. The hospital in a particular region being assigned to the doctor is specified in the contract and a region may choose a particular doctor for two separate hospitals. The two most important practical applications of the matching with contracts framework have used two different sufficient conditions. This paper provides a synthesis for the two conditions being used by proving that one is implied by the other.

The rest of the paper is organized as follows. Section 2 introduces the preliminaries of the many-to-one matching market with contracts. Section 3 gives the theorem, and a constructive proof using an algorithm. Section 4 gives an example with the details of the algorithm. Section 5 discusses the properties of stable matches. In the last section, I summarize the contribution of this paper.

\section{Preliminaries}

We use the model from Hatfield and Milgrom [2005], and Hatfield and Kojima [2010] and extend the notation from Kominers and Sönmez [2013]. A many-to-one matching market with contracts is parametrized by a finite set of doctors, $D$, a finite set of hospitals, $H$, and a finite set of contracts, $X$.

Each contract $x \in X$ is associated with a doctor $\mathrm{d}(x) \in D$ and a hospital $\mathrm{h}(x) \in$ $H$. We extend this notation to sets of contracts by defining $\mathrm{d}\left(X^{\prime}\right) \equiv \cup_{x \in X^{\prime}}\{\mathrm{d}(x)\}$ and $\mathrm{h}\left(X^{\prime}\right) \equiv \cup_{x \in X^{\prime}}\{\mathrm{h}(x)\}$ for $X^{\prime} \subseteq X$.

Definition 2.1. For a doctor $d$, a hospital $h$ and a set of contracts $X^{\prime}$, the subset of contracts with only a specific agent are defined as follows.

$$
\begin{aligned}
& X_{d}^{\prime} \equiv\left\{x \in X^{\prime} \mid \mathrm{d}(x)=d\right\} \\
& X_{h}^{\prime} \equiv\left\{x \in X^{\prime} \mid \mathrm{h}(x)=h\right\}
\end{aligned}
$$

Each doctor $d$ has access to a $\varnothing_{d}$ contract which represents being not matched to anybody. We say that $X^{\prime} \subseteq X$ is feasible for a hospital $h$ if $X^{\prime} \subseteq X_{h}$ and for all $x, x^{\prime} \in X^{\prime}$, we have $\mathrm{d}(x)=\mathrm{d}\left(x^{\prime}\right)$ implies $x=x^{\prime}$. A set of contracts $X^{\prime} \subseteq X$ is called an allocation if $x, x^{\prime} \in X^{\prime}$ and $\mathrm{d}(x)=\mathrm{d}\left(x^{\prime}\right)$ implies $x=x^{\prime}$. These conditions ensure that each doctor can sign at most one contract.

\footnotetext{
${ }^{6}$ More precisely, Kamada and Kojima [2015a] Theorem 2 follows directly from Kamada and Kojima [2015b] Theorem 1 which they prove using (many-to-many) matching with contracts framework.
} 


\subsection{Preferences of hospitals and doctors}

For each $d \in D, P_{d}$ is a strict preference relation on contracts in $X_{d} \cup\left\{\varnothing_{d}\right\}$. For each $h \in H, P_{h}$ is a strict preference relation on all feasible subsets of contracts of $X$ (including the null set). The primitives of this model are preferences over contracts or sets of contracts (and not choice functions over subsets). Hence, the Aygün and Sönmez [2013a,b]'s irrelevance of rejected contracts (IRC) condition holds in our setting. ${ }^{7}$ The IRC condition requires that the removal of rejected contracts should not affect the chosen set of contracts.

Definition 2.2. Given a set of contracts $X$, a choice function $C: 2^{X} \rightarrow 2^{X}$ satisfies the irrelevance of rejected contracts (IRC) condition if for all $Y \subset X$ and for all $z \in X \backslash Y$, we have $z \notin C(Y \cup\{z\})$ implies $C(Y)=C(Y \cup\{z\})$.

We define a choice function $C_{d}\left(X^{\prime}\right)$ (and $C_{h}\left(X^{\prime}\right)$ ) based on the strict preference ordering $P_{d}$ (and $P_{h}$ respectively). The choice function $C_{d}\left(X^{\prime}\right)$ will be a singleton that represents the chosen contract (possibly $\varnothing_{d}$ ) by the doctor $d$ from $X^{\prime} \cup\left\{\varnothing_{d}\right\}$. Similarly, $C_{h}\left(X^{\prime}\right)$ will represent the chosen set of contracts (possibly $\varnothing$ ) by the hospital $h$ from $X^{\prime}$ based on its strict preference ordering $P_{h}$ over its feasible subsets of contracts. Formally, for any set $X^{\prime} \subseteq X, d \in D$, and $h \in H$ let

$$
\begin{aligned}
C_{d}\left(X^{\prime}\right) & \equiv \max _{P_{d}}\left\{x \in X^{\prime} \mid d=\mathrm{d}(x)\right\} \cup\left\{\varnothing_{d}\right\} \\
C_{h}\left(X^{\prime}\right) & \equiv \max _{P_{h}}\left\{Z \subseteq X^{\prime} \mid x \in Z \Rightarrow h=\mathrm{h}(x)\right\}
\end{aligned}
$$

The rejection sets are defined as the contracts not chosen by the agents, i.e. $R_{d}\left(X^{\prime}\right) \equiv X^{\prime} \backslash\left\{C_{d}\left(X^{\prime}\right)\right\}$ and $R_{h}\left(X^{\prime}\right) \equiv X^{\prime} \backslash C_{h}\left(X^{\prime}\right)$. Further, the chosen set for all doctors is defined as the union of the chosen sets for each of the doctors, i.e. $C_{D}\left(X^{\prime}\right)=\cup_{d \in D} C_{d}\left(X^{\prime}\right)$. Likewise, we define the chosen sets for all hospitals, i.e. $C_{H}\left(X^{\prime}\right)=\cup_{h \in H} C_{h}\left(X^{\prime}\right)$.

\subsection{Stability and previous results}

Definition 2.3. An allocation $X^{\prime} \subseteq X$ is a stable allocation (or a stable set of contracts) if

1. (Individual Rationality) $C_{D}\left(X^{\prime}\right)=C_{H}\left(X^{\prime}\right)=X^{\prime}$, and

\footnotetext{
${ }^{7}$ When the primitives are choice functions instead of preference orders over sets of contracts, then this condition is not automatically guaranteed. See Aygün and Sönmez [2013b] for an illuminating discussion.
} 
2. (No Blocking) there does not exist a hospital $h$ and a set of contracts $X^{\prime \prime} \neq$ $C_{h}\left(X^{\prime}\right)$ such that $X^{\prime \prime}=C_{h}\left(X^{\prime} \cup X^{\prime \prime}\right) \subset C_{D}\left(X^{\prime} \cup X^{\prime \prime}\right)$.

Kelso and Crawford [1982] and Hatfield and Milgrom [2005] showed that the substitutability condition is sufficient for stability.

Definition 2.4. Contracts are substitutes for a hospital $h \in H$ if $\forall X^{\prime \prime} \subseteq X^{\prime} \subseteq X$, the rejection sets are isotone, i.e. $R_{h}\left(X^{\prime \prime}\right) \subseteq R_{h}\left(X^{\prime}\right)$. In other words, contracts are substitutes for a hospital $h \in H$ if for all contracts $x, z \in X$ and all sets $Y \subseteq X$, we have $z \in C_{h}(Y \cup\{z, x\})$ implies $z \in C_{h}(Y \cup\{z\}){ }^{8}$

Definition 2.5. A tuple $(x, z, Y)$ where $x, z \in X$ and $Y \subseteq X$, that fails the substitutes requirement, i.e. $z \in C_{h}(Y \cup\{z, x\})$ but $z \notin C_{h}(Y \cup\{z\})$, is called a substitutability violation. ${ }^{9}$ The contract $x$ in this case is called an alterer contract and the contract $z$ is termed as a recalled contract. ${ }^{10}$

The hospital preferences satisfy the substitutability condition if there are no substitutability violations. By permitting some specific violations, Hatfield and Kojima [2010] arrived at the following weaker conditions, which they proved to be sufficient for stability. Preferences are unilaterally substitutable if the only substitutability violations, $(x, z, Y)$, that exist are with sets $Y$ such that $\mathrm{d}(z) \in \mathrm{d}(Y)$, i.e. the doctor involved in the recalled contract has at least some contract in the set $Y$. The bilateral substitutability condition is weaker than the unilateral substitutability condition and the permissible substitutability violations, $(x, z, Y)$, are with sets $Y$ such that $\mathrm{d}(x) \in \mathrm{d}(Y)$ or $\mathrm{d}(z) \in \mathrm{d}(Y)$, i.e. either the doctor involved in the alterer contract or the recalled contract has some contract in the set $\mathrm{Y}$.

Definition 2.6. Contracts are unilateral substitutes for a hospital $h \in H$ if for all contracts $x, z \in X$ and all sets $Y \subseteq X$ such that $\mathrm{d}(z) \notin \mathrm{d}(Y)$, we have $z \in C_{h}(Y \cup\{z, x\})$ implies $z \in C_{h}(Y \cup\{z\}) .{ }^{11}$

Remark 2.1. If $(x, z, Y)$ is a substitutability violation in a preference which has the unilateral substitutability property then $\mathrm{d}(z) \in \mathrm{d}(Y \backslash\{z\})$.

\footnotetext{
${ }^{8}$ The requirement specified has a bite only when $x \in C_{h}(Y \cup\{z, x\})$ otherwise the requirement of $\left[z \in C_{h}(Y \cup\{z, x\}) \Rightarrow z \in C_{h}(Y \cup\{z\})\right]$ trivially holds due to IRC (definition 2.2).

${ }^{9}$ The definition was introduced and named as such in Hatfield, Immorlica, and Kominers [2012].

${ }^{10}$ The following preference $P_{h}$ fails the substitutability condition where $\mathrm{d}(z)=\mathrm{d}\left(z^{\prime}\right) \neq \mathrm{d}(x)$ and all contracts are with hospital $h . P_{h}:\{x, z\} \succ\{x\} \succ\left\{z^{\prime}\right\} \succ\{z\}$ as $\left(x, z,\left\{z^{\prime}\right\}\right)$ is a substitutability violation. $x$ is the alterer contract and $z$ is the recalled contract.

${ }^{11}$ The requirement specified has a bite only when $x \in C_{h}(Y \cup\{z, x\})$. See footnote 8
} 
Note that the definition of unilateral substitutability explicitly requires that for all the substitutability violations we should have $\mathrm{d}(z) \in \mathrm{d}(Y)$. The above remark which is stronger than the explicit requirement implies that for any substitutability violation $(x, z, Y)$ in unilaterally substitutable preferences, the doctor involved with the recalled contract has at least some contract other than $z$ in $Y$. If this were not true, i.e. suppose $\mathrm{d}(z) \notin \mathrm{d}(Y \backslash\{z\})$ then we will get a contradiction to the unilateral substitutability property. Define $\tilde{Y} \equiv Y \backslash\{z\}$ and it follows that $z \in$ $C_{h}(Y \cup\{z, x\})=C_{h}(\tilde{Y} \cup\{z, x\})$ and $z \notin C_{h}(Y \cup\{z\})=C_{h}(\tilde{Y} \cup\{z\})$. Thus $(x, z, \tilde{Y})$ is a substitutability violation and by assumption $\mathrm{d}(z) \notin \mathrm{d}(\tilde{Y})$. This would imply the contradiction.

Definition 2.7. Contracts are bilateral substitutes for a hospital $h \in H$ if for all contracts $x, z \in X$ and all sets $Y \subseteq X$ such that $\mathrm{d}(x), \mathrm{d}(z) \notin \mathrm{d}(Y)$, we have $z \in C_{h}(Y \cup\{z, x\})$ implies $z \in C_{h}(Y \cup\{z\}) .^{12}$

Remark 2.2. If $(x, z, Y)$ is a substitutability violation in a preference which satisfies bilateral substitutability property then $\mathrm{d}(z) \in \mathrm{d}(Y \backslash\{z\})$ or $\mathrm{d}(x) \in \mathrm{d}(Y \backslash\{x\})$.

The above remark which is stronger than the explicit requirement of the definition implies that for any substitutability violation, $(x, z, Y)$, in bilaterally substitutable preferences, the doctor involved with the recalled contract has at least some contract other than $z$ in $Y$ or the doctor involved with the alterer contract has at least some contract other than $x$ in $Y$. This holds for exactly the same reasons as in Remark 2.1 above.

Hatfield and Kominers [2014] provided a condition termed substitutable completability as a sufficient condition for the existence of a stable matching. They use the many-to-many matching markets setup and preferences to give this sufficient condition.

Definition 2.8. A many-to-many preference for a hospital $h$ is a preference relation over all subsets $X^{\prime} \subseteq X$ such that $X_{h}^{\prime}=X^{\prime} .{ }^{13}$

The process of preference completion involves adding infeasible sets that include multiple contracts with some doctors, to a many-to-one preference relation (which is only over feasible sets of contracts).

Definition 2.9. A completion of a many-to-one choice function $C_{h}$, corresponding to the preference $P_{h}$ for a hospital $h \in H$, is a choice function $\bar{C}^{h}$ such that for all $Y \subseteq X$ either

\footnotetext{
${ }^{12}$ The requirement specified has a bite only when $x \in C_{h}(Y \cup\{z, x\})$. See footnote 8

${ }^{13} \mathrm{~A}$ preference profile in the many-to-one matching market with contracts was over all feasible subsets $X^{\prime} \subseteq X_{h}$ where as the many-to-many preference is over all subsets $X^{\prime} \subseteq X_{h}$.
} 
1. $\bar{C}_{h}(Y)=C_{h}(Y)$ or

2. $\exists$ distinct $z, \hat{z} \in \bar{C}_{h}(Y)$ such that $\mathrm{d}(z)=\mathrm{d}(\hat{z})$

If a choice function $C_{h}$ has a completion that is substitutable and satisfies the IRC condition, then we say that the $C_{h}$ is substitutably completable.

In our setting where we take the preference $P_{h}$ as the primitive for the hospitals, the equivalent definition of substitutable completability is as follows.

Definition 2.10. A preference $P_{h}$ is substitutably completable for hospital $h \in H$ if there exists a substitutable many-to-many preference $\bar{P}_{h}$ such that $\bar{P}_{h}$ satisfies the following conditions:

- For feasible $X^{\prime}, X^{\prime \prime} \subseteq X$, if $X^{\prime} \succ_{h} X^{\prime \prime} \succ_{h} \varnothing$ under $P_{h}$ then $X^{\prime} \succ_{h} X^{\prime \prime} \succ_{h} \varnothing$ under $\bar{P}_{h}$.

- For feasible $X^{\prime} \subseteq X$, if $\varnothing \succ_{h} X^{\prime}$ under $P_{h}$ then it remains unacceptable, i.e. $\varnothing \succ_{h} X^{\prime}$ under $\overline{\bar{P}}_{h}$.

$\bar{P}_{h}$ is defined as a substitutable completion of the preference $P_{h}$.

Since the resulting completion is substitutable, there exists a stable matching in the many-to-many setting due to the sufficiency of the substitutability condition (Hatfield and Kominers [2012, 2013]). This stable matching also respects the restrictions of the many-to-one setting, as the preferences for the doctors were not changed and they still find only singleton contracts acceptable. ${ }^{14}$

\section{Main Result}

We now present the main result of this paper. We provide the intuition behind the constructive proof and describe the algorithm before proceeding to the proof of this theorem.

Theorem 1. If the hospital preferences are unilaterally substitutable then they are substitutably completable, i.e. for any $P_{h}$ with the unilateral substitutability property, there exists a completion $\bar{P}_{h}$ which satisfies the substitutability property.

\footnotetext{
${ }^{14} \mathrm{~A}$ stable match in the many-to-many setting should be individually rational for each doctor which rules out a subset of contracts that is not an allocation.
} 
We now present the main idea behind how the constructive proof achieves a substitutable completion of preferences. Recall that a preference has the substitutability property if and only if

- there are no substitutability violations.

Further recall that a preference is unilaterally substitutable if and only if

- there are no substitutability violations OR

- there are only 'permissible' substitutability violations.

Specifically, all violations $(x, z, Y)$ that exist are the permissible ones where the doctor of the recalled contract, i.e. $\mathrm{d}(z)$, has some contract in $Y$ (other than $z) .{ }^{15}$ For example, consider the following preferences $P_{h}$ for a hospital $h$. Here we have, $\mathrm{d}(z)=\mathrm{d}\left(z^{\prime}\right) \neq \mathrm{d}(x)$ and all the contracts listed are with hospital $h$.

$$
\begin{aligned}
P_{h}: & \{x, z\} \succ\{x\} \succ\left\{z^{\prime}\right\} \succ\{z\} \succ \varnothing \\
\left(x, z,\left\{z^{\prime}\right\}\right) \quad & \text { is the only substitutability violation } \\
& \text { and it is permissible. }
\end{aligned}
$$

The algorithm uses the recalled contract and the existing contract(s) with $\mathrm{d}(z)$ in set $Y$ and adds them to the chosen set of contracts under the original preferences from $Y \cup\{z\}$ to create the new chosen set under the new preference. The change in the chosen set is achieved by making the new chosen set (just) better than the older chosen set. The presence of at least two contracts with $\mathrm{d}(z)$ ensures that the new chosen set being added in the preference relation is an infeasible set. This ensures that the resulting new preference is a completion. To ensure that the completion process does not create any new substitutability violations while correcting the existing ones, the algorithm may also change a chosen set that includes a contract involving $\mathrm{d}(z)$ under the original preference. This further ensures that the final completion created by the algorithm is substitutable. The following example elucidates this process.

$$
\begin{aligned}
C_{h}\left(\left\{z, z^{\prime}\right\}\right)= & \left\{z^{\prime}\right\} \text { as per the preference relation } P_{h} \\
\hat{C}_{h}\left(\left\{z, z^{\prime}\right\}\right)= & \left\{z, z^{\prime}\right\} \text { by completing the preference to } \bar{P}_{h} \\
\bar{P}_{h}: & \{x, z\} \succ\{x\} \succ\left\{z^{\prime}, z\right\} \succ\left\{z^{\prime}\right\} \succ\{z\} \succ \varnothing \\
\left(x, z,\left\{z^{\prime}\right\}\right) \quad & \text { is not a substitutability violation }
\end{aligned}
$$

\footnotetext{
${ }^{15}$ See remark 2.1 .
} 
Before proceeding to the proof, we introduce the following concept which will be used in one of the steps of the algorithm, and provide an example which clarifies the relevance of this concept.

Definition 3.1. The maximal subset of $\tilde{Y}$ with only unrejected contracts with a doctor, say $d_{1}$, under a preference $P_{h}$, is defined as the set $\hat{Y} \subseteq \tilde{Y}$ such that

1. All non-d $d_{1}$ contracts of $\tilde{Y}$ are present in $\hat{Y}$, i.e. $\hat{Y} \backslash \hat{Y}_{d_{1}}=\tilde{Y} \backslash \tilde{Y}_{d_{1}}$

2. $\forall Y^{\prime \prime} \subseteq \hat{Y}, d_{1} \in \mathrm{d}\left(Y^{\prime \prime}\right) \quad \Rightarrow \quad d_{1} \in \mathrm{d}\left(C_{h}\left(Y^{\prime \prime}\right)\right)$

and $\hat{Y}$ is the maximal such subset under the partial order of set inclusion. ${ }^{16}$

Note the maximal set defined above is unique due to the many-to-one nature of the setting. ${ }^{17}$ This maximal subset only has contracts which are, loosely speaking, not always rejected in the original preference. Consider the following preference which will explain the above concept.

\section{Example 3.1.}

$$
\begin{aligned}
& P_{h}:\{x, z\} \succ\{x\} \succ\left\{z^{\prime}\right\} \succ\{z\} \succ \varnothing \succ\{\hat{z}\} \\
& \mathrm{d}(z)=\mathrm{d}\left(z^{\prime}\right)=\mathrm{d}(\hat{z}) \neq \mathrm{d}(x)
\end{aligned}
$$

Let $\tilde{Y}=\left\{z^{\prime}, z, \hat{z}\right\}$. The maximal subset of $\tilde{Y}$ with only unrejected contracts with doctor $d_{1}=\mathrm{d}(\hat{z})$ under $P_{h}$ is $\hat{Y}=\left\{z^{\prime}, z\right\}$ because $\mathrm{d}(\hat{z}) \notin \mathrm{d}\left(C_{h}(\{\hat{z}\})\right)$.

Now we are ready to present the algorithm with the understanding of the underlying intuition presented above.

\subsection{Algorithm}

Step 0 Define $P_{h}^{0} \equiv P_{h}$ and $C_{h}^{0}(\cdot) \equiv C_{h}(\cdot)$ for the hospital $h$. Go to step 1 .

Step $i$ Does there exist a substitutability violation $\left(x_{i}, z_{i}, Y_{i}\right)$ for $P_{h}^{i-1} ?^{18}$

- If yes define $C_{h}^{i}(\tilde{Y})$ as follows for all $\tilde{Y} \subseteq X_{h}$.

$$
\text { * If } \mathrm{d}\left(z_{i}\right) \notin \mathrm{d}\left(C_{h}^{i-1}[\tilde{Y}]\right) \text { then } C_{h}^{i}(\tilde{Y}) \equiv C_{h}^{i-1}(\tilde{Y}) \text {. }
$$

\footnotetext{
${ }^{16}$ Maximality of $\hat{Y}$ implies that $\nexists$ any other set $\tilde{\tilde{Y}} \subseteq \tilde{Y}$ but not a subset of $\hat{Y}$ for which the following conditions holds, $\tilde{\tilde{Y}} \backslash[\tilde{\tilde{Y}}]_{d_{1}}=\tilde{Y} \backslash \tilde{Y}_{d_{1}}$ and $\forall Y^{\prime \prime} \subseteq \tilde{\tilde{Y}}, d_{1} \in \mathrm{d}\left(Y^{\prime \prime}\right) \Rightarrow d_{1} \in \mathrm{d}\left[C_{h}\left(Y^{\prime \prime}\right)\right]$

${ }^{17}$ See Appendix Claim 1 for the proof.

${ }^{18}$ Without loss of generality, we can restrict our attention to $x_{i}, z_{i} \in X_{h}$ and $Y_{i} \subseteq X_{h}$.
} 
* If $\mathrm{d}\left(z_{i}\right) \in \mathrm{d}\left(C_{h}^{i-1}[\tilde{Y}]\right)$ then find the maximal subset $\hat{Y}$ of the set under consideration, $\tilde{Y}$ with only unrejected contracts with doctor $\mathrm{d}\left(z_{i}\right)$ under $P_{h}^{i-1}$. Define the choice function as $C_{h}^{i}(\tilde{Y}) \equiv C_{h}^{i-1}(\tilde{Y}) \cup[\hat{Y}]_{\mathrm{d}\left(z_{i}\right)}$.

The choice function $C_{h}^{i}$ has the underlying preference relation $P_{h}^{i}$. The existence of such a preference relation over subsets of $X_{h}$ is proved in Claim 2 of the Appendix with a constructive proof.

Go to step $i+1$.

- If not then $I \equiv i-1$ and $\bar{P}_{h} \equiv P_{h}^{I}$ and terminate the algorithm. ${ }^{19}$

We start with the following four observations about the algorithm which will be useful at various steps of the proofs that will follow.

1. During step $i$, preferences are changed to $P_{h}^{i}$ only if a substitutability violation $\left(x_{i}, z_{i}, Y_{i}\right)$ is identified in $P_{h}^{i-1}$.

2. The chosen sets are weakly increasing in the order of set inclusion at all steps of the algorithm. Specifically, for all $\tilde{Y} C_{h}^{i-1}(\tilde{Y}) \subseteq C_{h}^{i}(\tilde{Y})$. We refer to this property as the property of a weak order over the chosen sets.

3. The chosen set $C_{h}^{i}(\tilde{Y})$ could be different from $C_{h}^{i-1}(\tilde{Y})$ only if $\mathrm{d}\left(z_{i}\right) \in \mathrm{d}\left(C_{h}^{i-1}(\tilde{Y})\right)$.

4. Moreover, $C_{h}^{i}(\tilde{Y})$ could be different from $C_{h}^{i-1}(\tilde{Y})$ only in contracts with doctor $\mathrm{d}\left(z_{i}\right)$.

We use $C_{h}^{i}(\cdot)$ and $P_{h}^{i}$ interchangeably, above and for the rest of the proof, as may be appropriate for the context. The existence of $P_{h}^{i}$ is guaranteed in the algorithm as proved in Claim 2 of the Appendix.

\subsection{Lemmas}

We define a new violation as a substitutability violation that exists in $P_{h}^{i}$ but not in $P_{h}^{i-1}$ for some $i \geq 1$ in the above algorithm. We now prove that no such new violations are created by the algorithm.

Lemma 3.1. No new violations are created in the algorithm above when preferences of hospitals satisfy the irrelevance of rejected contracts condition.

\footnotetext{
${ }^{19}$ Section 4 has an illustrative example worked out.
} 
Proof We prove this by contradiction. Suppose there are new violations introduced in the algorithm above. Let us consider the first step ' $i$ ' where a new violation, $(x, z, Y)$ is introduced. We have $z \in C_{h}^{i}(Y \cup\{x, z\})$ but $z \notin C_{h}^{i}(Y \cup\{z\})$. We then prove the following.

(I) The contract $z$ is added to the chosen set of $Y \cup\{x, z\}$ in step $i$, i.e. $z \notin$ $C_{h}^{i-1}(Y \cup\{x, z\})$ and $z \in C_{h}^{i}(Y \cup\{x, z\})$.

(II) The doctor $\mathrm{d}(z)$ has some contract in the chosen set from $Y \cup\{z\}$ under $P_{h}^{i-1}$.

(III) The contract $z$ is a part of the chosen set under $P_{h}^{i}$ of $Y \cup\{z\}$ at step $i$ which contradicts the assumption that $(x, z, Y)$ is a substitutability violation.

(I) To prove the first claim recall that $z \in C_{h}^{i}(Y \cup\{x, z\})$ but $z \notin C_{h}^{i}(Y \cup\{z\})$. Along with observation 2 about the weak order over the chosen sets, this implies that $z \notin C_{h}^{i-1}(Y \cup\{z\})$.

As $(x, z, Y)$ is not a violation in step $i-1$, we necessarily have $z \notin C_{h}^{i-1}(Y \cup$ $\{x, z\}){ }^{20}$ Thus the chosen set from $Y \cup\{x, z\}$ is modified during step $i$.

We can further claim that $z$ is added to the chosen set from $Y \cup\{x, z\}$. Let $\hat{Y}_{1}$ be the maximal subset of $Y \cup\{x, z\}$ as described in definition 3.1. From the algorithm above and observations 1, 2, and 4, we know that step $i$ identified a substitutability violation $\left(x_{i}, z_{i}, Y_{i}\right)$ and we have the following.

$$
\mathrm{d}(z)=\mathrm{d}\left(z_{i}\right) \text { and } z \in \hat{Y}_{1}
$$

(II) We prove the second claim by contradiction. Suppose not, i.e. $\mathrm{d}(z) \notin$ $\mathrm{d}\left[C_{h}^{i-1}(Y \cup\{z\})\right]$. We have maintained the assumption that the primitives in this setting are preferences over contracts and not choice correspondences, which guarantees the IRC condition.

Define $\tilde{Y}=Y \backslash Y_{\mathrm{d}(z)} \cup\{z\}$. By IRC, we have the following.

$$
\mathrm{d}(z) \notin \mathrm{d}\left[C_{h}^{i-1}(\tilde{Y})\right]
$$

Recall that the maximal subset $\hat{Y}_{1}$ of $Y \cup\{x, z\}$ satisfies the following properties.

(a) $\hat{Y}_{1} \backslash\left[\hat{Y}_{1}\right]_{\mathrm{d}(z)}=Y \cup\{x, z\} \backslash[Y \cup\{x, z\}]_{\mathrm{d}(z)}=Y \cup\{x\} \backslash Y_{\mathrm{d}(z)}$

(b) $\forall Y_{1}^{\prime \prime} \subseteq \hat{Y}_{1}, \mathrm{~d}(z) \in \mathrm{d}\left(Y_{1}^{\prime \prime}\right) \Rightarrow \mathrm{d}(z) \in \mathrm{d}\left[C_{h}\left(Y_{1}^{\prime \prime}\right)\right]$

\footnotetext{
${ }^{20}$ We know that $z \in C_{h}^{i-1}(Y \cup\{x, z\}) \Rightarrow z \in C_{h}^{i-1}(Y \cup\{z\})$ is true.
} 
We can make the following set of conclusions using the definition of $\tilde{Y}$ above and the two conditions on $\hat{Y}_{1}$.

$$
\begin{aligned}
& \tilde{Y} \backslash\{z\} \subseteq \hat{Y}_{1} \backslash\left[\hat{Y}_{1}\right]_{\mathrm{d}(z)} \\
& z \in \hat{Y}_{1} \text { therefore } \tilde{Y} \subseteq \hat{Y}_{1} \\
& \text { Since } \mathrm{d}(z) \in \mathrm{d}(\tilde{Y}) ; \mathrm{d}(z) \in \mathrm{d}\left[C_{h}(\tilde{Y})\right]
\end{aligned}
$$

However, this is at odds with equation 4 above and observation 2. This leads to the required contradiction. Hence we have $\mathrm{d}(z) \in \mathrm{d}\left[C_{h}^{i-1}(Y \cup\{z\})\right]$.

(III) Let the maximal subset of $Y \cup\{z\}$ as per definition 3.1 be $\hat{Y}_{2}$. Since d $(z) \in$ $\mathrm{d}\left[C_{h}^{i-1}(Y \cup\{z\})\right]$, the chosen set is (possibly) altered at step $i$ using $\hat{Y}_{2}$. We want to prove that $z \in \hat{Y}_{2}$. Suppose not. Then $\hat{Y}_{2} \cup\{z\}$ is a larger set than $\hat{Y}_{2}$ but is not the required maximal subset. Let us observe that all non-d $(z)$ contracts of $Y \cup\{z\}$ are present in $\hat{Y}_{2}$ and consequently in $\hat{Y}_{2} \cup\{z\}$ as well. Hence $\hat{Y}_{2} \cup\{z\}$ must fail the second requirement of the maximal subset. There must exist a set $Y_{2}^{\prime \prime} \subseteq \hat{Y}_{2} \cup\{z\}$ such that the following is true.

$$
\mathrm{d}(z) \in \mathrm{d}\left(Y_{2}^{\prime \prime}\right) \text { but } \mathrm{d}(z) \notin \mathrm{d}\left[C_{h}\left(Y_{2}^{\prime \prime}\right)\right]
$$

However, $Y_{2}^{\prime \prime} \nsubseteq \hat{Y}_{2}$ otherwise that would be a violation of the second requirement for the maximal subset, $\hat{Y}_{2}$ in definition 3.1. This implies that $z \in Y_{2}^{\prime \prime}$.

Define $\tilde{Y}_{2}=Y_{2}^{\prime \prime} \backslash\left[Y_{2}^{\prime \prime}\right]_{\mathrm{d}(z)} \cup\{z\}$. Clearly $z \in \tilde{Y}_{2}$ and from 5 and IRC we have,

$$
\mathrm{d}(z) \notin\left[C_{h}\left(\tilde{Y}_{2}\right)\right]
$$

Recall that $\hat{Y}_{1}$ is the maximal subset of $Y \cup\{x, z\}$. We can compare $\tilde{Y}_{2}$ with $\hat{Y}_{1}$ and $\hat{Y}_{2}$ more closely.

$$
\begin{aligned}
\tilde{Y}_{2} \backslash\{z\} & =Y_{2}^{\prime \prime} \backslash\left[Y_{2}^{\prime \prime}\right]_{\mathrm{d}(z)} \\
& \subseteq \hat{Y}_{2} \backslash\left[\hat{Y}_{2}\right]_{\mathrm{d}(z)}=Y \backslash Y_{\mathrm{d}(z)} \\
& \subseteq Y \cup\{x\} \backslash Y_{\mathrm{d}(z)}=\hat{Y}_{1} \backslash\left[\hat{Y}_{1}\right]_{\mathrm{d}(z)} \subseteq \hat{Y}_{1}
\end{aligned}
$$

We have $\tilde{Y}_{2} \subseteq \hat{Y}_{1} \cup\{z\}=\hat{Y}_{1}$ (from equation 3 ).

Since we have $z \in \tilde{Y}_{2}$ by the second condition on the maximal subset, $\hat{Y}_{1}$ we have $\mathrm{d}(z) \in\left[C_{h}\left(\tilde{Y}_{2}\right)\right]$ which contradicts the conclusion above in equation 6 .

Hence we have $z \in \hat{Y}_{2}$ and thus $z$ is a part of the chosen set from $Y \cup\{z\}$ at step i, i.e. $z \in C_{h}^{i}[Y \cup\{z\}]$.

This provides the required contradiction to $(x, z, Y)$ being a substitutability violation. 
An immediate corollary is that the unilateral substitutability property is preserved at each step in the algorithm above. Otherwise, there would be a new violation in $P_{i}$ that was not present in the original preference $P_{h}$. This would contradict the lemma.

Corollary 3.1. The preference $P_{h}^{i}$ created in the algorithm above at each step $i$ has the unilateral substitutability property if $P_{h}$ is unilaterally substitutable.

The next lemma shows that the algorithm eliminates the substitutability violations one by one.

Lemma 3.2. Suppose preferences satisfy the unilateral substitutability condition. For each step $i$, the substitutability violation $\left(x_{i}, z_{i}, Y_{i}\right)$ under $P_{h}^{i-1}$ is corrected and is not a violation under $P_{h}^{i}$.

Proof We prove this in two steps. In the first part, we establish that for the substitutability violation $\left(x_{i}, z_{i}, Y_{i}\right), \exists \hat{z}_{i} \in C_{h}^{i-1}\left(Y_{i} \cup\left\{z_{i}\right\}\right)$ such that $\mathrm{d}\left(\hat{z}_{i}\right)=\mathrm{d}\left(z_{i}\right)$. In the second part, we prove that $z_{i}$ is added to the chosen set from $Y_{i} \cup\left\{z_{i}\right\}$ in step $i$ and thus the violation is fixed. ${ }^{21}$

Part I By Lemma 3.1, we know that the violation considered in step $i$ also existed in the original preferences $P_{h}$. Further note that $z_{i} \in C_{h}\left(Y_{i} \cup\left\{z_{i}, x_{i}\right\}\right)$ and hence there does not exist any other $\mathrm{d}\left(z_{i}\right)$ contract in $C_{h}\left(Y_{i} \cup\left\{z_{i}, x_{i}\right\}\right)$ as the original preferences were defined only over feasible subsets of contracts. Using irrelevance of rejected contracts, we will have $C_{h}\left(Y_{i} \cup\left\{z_{i}, x_{i}\right\}\right)=C_{h}\left(Y_{i} \backslash\left[Y_{i}\right]_{\mathrm{d}\left(z_{i}\right)} \cup\left\{z_{i}, x_{i}\right\}\right)$ where $\left[Y_{i}\right]_{\mathrm{d}\left(z_{i}\right)}$ is the set of contracts in $Y_{i}$ involving doctor $\mathrm{d}\left(z_{i}\right)$.

If $\nexists \hat{z}_{i}{ }^{1} \in C_{h}\left(Y_{i} \cup\left\{z_{i}\right\}\right)$ such that $\mathrm{d}\left(\hat{z}_{i}{ }^{1}\right)=\mathrm{d}\left(z_{i}\right)$ then $C_{h}\left(Y_{i} \cup\left\{z_{i}\right\}\right)=C_{h}\left(Y_{i} \backslash\right.$ $\left.\left[Y_{i}\right]_{\mathrm{d}\left(z_{i}\right)} \cup\left\{z_{i}\right\}\right)$. Define $\hat{Y}=Y_{i} \backslash\left[Y_{i}\right]_{\mathrm{d}\left(z_{i}\right)}$ and we have

$$
\begin{array}{ll}
z_{i} & \notin C_{h}\left(\hat{Y} \cup\left\{z_{i}\right\}\right) \\
z_{i} & \in C_{h}\left(\hat{Y} \cup\left\{z_{i}, x_{i}\right\}\right)
\end{array}
$$

$\left(x_{i}, z_{i}, \hat{Y}\right)$ is a substitutability violation of $P_{h}$ and $\mathrm{d}\left(z_{i}\right) \notin \mathrm{d}(\hat{Y})$ which implies the preferences do not satisfy unilateral substitutability condition. Hence, our initial assumption must be incorrect and in fact, $\mathrm{d}\left(z_{i}\right) \in \mathrm{d}\left(C_{h}\left(Y_{i} \cup\left\{z_{i}\right\}\right)\right)$. Moreover, by the weak order over the chosen sets there exists a $\hat{z}_{i} \in C_{h}^{i-1}\left(Y_{i} \cup\left\{z_{i}\right\}\right)$ such that $\mathrm{d}\left(\hat{z}_{i}\right)=\mathrm{d}\left(z_{i}\right)$.

\footnotetext{
${ }^{21}$ The claims in these two parts are very similar to those of steps (II) and (III) of Lemma 3.1 but the proof is very different because for the 'new' violation we knew that $z \in \hat{Y}_{1}$. Instead, here we have $z_{i} \in C_{h}\left(Y_{i} \cup\left\{x_{i}, z_{i}\right\}\right)$ but the unilateral substitutability condition helps us prove these claims.
} 
Part II Consider the maximal subset ${ }^{22} \hat{Y}$ of $Y_{i} \cup\left\{z_{i}\right\}$ that includes only the unrejected contracts with $\mathrm{d}\left(z_{i}\right)$. We prove that $z_{i} \in \hat{Y}$ by contradiction. Suppose not and we have $z_{i} \notin \hat{Y}$. Consider $\hat{Y} \cup\left\{z_{i}\right\}$ which contains all the non-d $\left(z_{i}\right)$ contracts of $Y_{i} \cup\left\{z_{i}\right\}$ but is not the required maximal subset. Hence it fails the second condition of the maximal subset.

$$
\begin{aligned}
& \exists \tilde{Y} \subseteq \hat{Y} \cup\{z\} \text { such that } \\
& \mathrm{d}\left(z_{i}\right) \in \tilde{Y} \text { but } \mathrm{d}\left(z_{i}\right) \notin \mathrm{d}\left[C_{h}^{i-1}(\tilde{Y})\right]
\end{aligned}
$$

We can conclude that $z_{i} \in \tilde{Y}$. If not, $\tilde{Y} \subseteq \hat{Y}$ and $\tilde{Y}$ will not be a (maximal) subset with only unrejected contracts with $\mathrm{d}\left(z_{i}\right)$. By the irrelevance of rejected contracts condition, there also exists $\tilde{Y}_{1} \cup\left\{z_{i}\right\} \subseteq \tilde{Y}$ with $\left[\tilde{Y}_{1}\right]_{\mathrm{d}\left(z_{i}\right)}=\emptyset$, i.e. the only d $\left(z_{i}\right)$ contract in $\tilde{Y}_{1} \cup\left\{z_{i}\right\}$ is $z_{i}$, satisfying $\mathrm{d}\left(z_{i}\right) \in \mathrm{d}\left(\tilde{Y}_{1} \cup\left\{z_{i}\right\}\right)$ but $\mathrm{d}\left(z_{i}\right) \notin \mathrm{d}\left[C_{h}^{i-1}\left(\tilde{Y}_{1} \cup\left\{z_{i}\right\}\right)\right]$.

Thus we have $\tilde{Y}_{1} \cup\left\{z_{i}\right\} \subseteq \tilde{Y} \subseteq \hat{Y} \cup\left\{z_{i}\right\} \subseteq Y_{i} \cup\left\{z_{i}\right\} \subseteq Y_{i} \cup\left\{z_{i}, x_{i}\right\}$. We also have the following.

$$
\begin{aligned}
z_{i} \in \tilde{Y}_{1} \cup\left\{z_{i}\right\} & \text { and } \quad z_{i} \notin C_{h}^{i-1}\left(\tilde{Y}_{1} \cup\left\{z_{i}\right\}\right) \\
z_{i} \in Y_{i} \cup\left\{z_{i}, x_{i}\right\} & \text { and } \quad z_{i} \in C_{h}^{i-1}\left(Y_{i} \cup\left\{z_{i}, x_{i}\right\}\right)
\end{aligned}
$$

By the weak order over the chosen sets, we also know that $z_{i} \notin C_{h}\left(\tilde{Y}_{1} \cup\left\{z_{i}\right\}\right)$ and since the violation existed in the original preferences $P_{h}, z_{i} \in C_{h}\left(Y_{i} \cup\left\{z_{i}, x_{i}\right\}\right)$. Moreover, we have that $\left[\tilde{Y}_{1}\right]_{\mathrm{d}\left(z_{i}\right)}=\emptyset$ and hence $\tilde{Y}_{1} \cup\left\{z_{i}\right\} \subseteq Y_{i} \backslash\left[Y_{i}\right]_{\mathrm{d}\left(z_{i}\right)} \cup\left\{z_{i}, x_{i}\right\}$. Thus we have the following.

$$
\begin{aligned}
z_{i} \in \tilde{Y}_{1} \cup\left\{z_{i}\right\} \quad \text { and } \quad & z_{i} \notin C_{h}\left(\tilde{Y}_{1} \cup\left\{z_{i}\right\}\right) \\
z_{i} \in Y_{i} \backslash\left[Y_{i}\right]_{\mathrm{d}\left(z_{i}\right)} \cup\left\{z_{i}, x_{i}\right\} \quad \text { and } \quad & z_{i} \in C_{h}\left(Y_{i} \backslash\left[Y_{i}\right]_{\mathrm{d}\left(z_{i}\right)} \cup\left\{z_{i}, x_{i}\right\}\right)
\end{aligned}
$$

Define $\tilde{\tilde{Y}} \equiv Y_{i} \backslash\left[Y_{i}\right]_{\mathrm{d}\left(z_{i}\right)} \cup\left\{z_{i}, x_{i}\right\}$. Label all the elements in $\tilde{\tilde{Y}} \backslash\left[\tilde{Y}_{1} \cup\left\{z_{i}\right\}\right]$ as $q_{1}, q_{2}, q_{3}, \ldots, q_{J}$. It is clear that $\mathrm{d}\left(q_{j}\right) \neq \mathrm{d}\left(z_{i}\right) \forall j \in\{1,2, \cdots, J\}$. Moreover, when we add one contract at a time to the set $\tilde{Y}_{1} \cup\left\{z_{i}\right\}$ to arrive at newer chosen sets, $z_{i}$ will become acceptable (eventually). There would be a first $\hat{\jmath}$ such that we have the following $z_{i} \in C_{h}\left(\tilde{Y}_{1} \cup\left\{q_{1}, q_{2}, \ldots, q_{\hat{\jmath}}\right\} \cup\left\{z_{i}\right\}\right)$. We found a substitutability violation $(x, z, Y)$ where $x=q_{\hat{\jmath}}, z=z_{i}$, and $Y=\tilde{Y}_{1} \cup\left\{q_{1}, q_{2}, \ldots, q_{\hat{\jmath}-1}\right\}$ where $\mathrm{d}(z) \notin \mathrm{d}(Y)$ and thus the original preference is not unilaterally substitutable. This is a contradiction and in fact we always have $z_{i} \in \hat{Y}$.

If $z_{i} \in \hat{Y}$ then $z_{i} \in C_{h}^{i}\left(Y_{i} \cup\left\{z_{i}\right\}\right)$ and the violation does not exist in $P_{h}^{i}$.

Lemma 3.3. $P_{h}^{i}$ is a completion of $P_{h} \forall i$.

\footnotetext{
${ }^{22}$ See Definition 3.1
} 
Proof For $i=0$ this is trivially true as each preference is a trivial completion of itself. For $i \geq 1$, we need to prove that in the algorithm, $P_{h}^{i}$ is modified from $P_{h}^{i-1}$ by including infeasible sets and not changing the order of feasible sets. If we use the choice function definition of the completion instead, then we need to prove that the chosen contracts by the hospital are either the same or have more than one (distinct) contracts with the same doctor. For any $\tilde{Y}$ such that $\mathrm{d}\left(z_{i}\right) \notin \mathrm{d}\left[C_{h}^{i-1}(\tilde{Y})\right]$, $C_{h}^{i}(\tilde{Y})=C_{h}^{i-1}(\tilde{Y})$. If $\tilde{Y}$ is such that $\mathrm{d}\left(z_{i}\right) \in \mathrm{d}\left[C_{h}^{i-1}(\tilde{Y})\right]$, then the corresponding maximal subset $\hat{Y}$ contains either one contract or more than one contracts involving $\mathrm{d}\left(z_{i}\right)$ and the hospital $h$. If the maximal subset contains only one contract, that contract will be a part of the chosen set in step $i-1$. Then essentially we have $C_{h}^{i}(\tilde{Y})=C_{h}^{i-1}(\tilde{Y})$. Moreover, if the maximal subset contains two or more contracts involving $\mathrm{d}\left(z_{i}\right)$ and the hospital $h$, all those contracts would be included in the new definition of $C_{h}^{i}(\tilde{Y})$. This ensures that there are at least two distinct contracts with the same doctor $\mathrm{d}\left(z_{i}\right)$ when $C_{h}^{i}(\tilde{Y}) \neq C_{h}^{i-1}(\tilde{Y})$.

Both these conditions imply that $C_{h}^{i}$ is a completion of $C_{h}^{i-1}$ and by induction on $i$, we can say that $C_{h}^{i}$ is a completion for all $\mathrm{i}$. The existence of a unique $P_{h}^{i}$ as guaranteed by Claim 2 in the appendix for each $C_{h}^{i}$ completes the proof of the claim above.

Now we are ready to give the proof of the main result.

Proof of Theorem 1 Since there is a finite number of contracts, for a given preference ordering for a hospital there is a finite number of (possible) substitutability violations. Since at each step no new violations are created (by Lemma 3.1) and at least one violation is reduced (by Lemma 3.2), the number of violations at each step is strictly less than the number of violations in the previous step. Since we had a finite number to begin with and it strictly decreases progressively, the algorithm is bound to end in finitely many steps. After the algorithm terminates, we have $P_{h}^{I}$ defined as $\bar{P}_{h}$. This preference has no violations and hence satisfies the substitutability condition. It is a completion of the original preference $P_{h}$ by Lemma 3.3. Hence it is a substitutable completion of the preferences $P_{h}$ with the unilateral substitutability property. Note that this property was needed to guarantee that Lemma 3.2 goes through. This ensured that the number of violations strictly decrease at each step which was crucial for this algorithm to work.

In the algorithm presented above at each step $i$, all the substitutability violations involving the recalled contract doctor, i.e. $\mathrm{d}(z)$, are fixed. This gives the following proposition about the number of steps in the algorithm above.

Proposition 3.1. If the hospital preferences are unilaterally substitutable then the completion algorithm identifies at most $|D|$ substitutability violations and thus com- 
pletes in at most $|D|$ steps.

There are at most $|D|$ doctors who would have a substitutability violation in a given hospital preference and hence the number of steps in the algorithm 3.1 is capped at the cardinality of the set of doctors. ${ }^{23}$ We present the proof of this in the appendix.

\section{Example}

I explain the algorithm above using an example. Consider the following preferences with unilateral substitutability property for a hospital $h$ over a set of contracts $X_{h}=$ $\left\{x, x^{\prime}, y, y^{\prime}\right\}$. We also have $\mathrm{d}(x)=\mathrm{d}\left(x^{\prime}\right) \neq \mathrm{d}(y)=\mathrm{d}\left(y^{\prime}\right)$.

$$
P_{h}:\left\{x, y^{\prime}\right\} \succ\left\{x^{\prime}, y\right\} \succ\left\{x^{\prime}, y^{\prime}\right\} \succ\{x, y\} \succ\left\{x^{\prime}\right\} \succ\left\{y^{\prime}\right\} \succ\{x\} \succ\{y\}
$$

The four violations are as follows, where the first contract denoted as $z$ in the discussion so far is rejected from the set $Y \cup\{z\}$ but becomes acceptable when a different contract $x$ becomes available as well.

\begin{tabular}{ccc}
$\mathrm{x}$ & $\mathrm{z}$ & $\mathrm{Y}$ \\
\hline $\mathrm{y}^{\prime}$ & $\mathrm{x}$ & $\left\{\mathrm{x}^{\prime}\right\}$ \\
$\mathrm{y}^{\prime}$ & $\mathrm{x}$ & $\left\{\mathrm{x}^{\prime}, \mathrm{y}\right\}$ \\
$\mathrm{x}^{\prime}$ & $\mathrm{y}$ & $\left\{\mathrm{y}^{\prime}\right\}$ \\
$\mathrm{x}$ & $\mathrm{y}^{\prime}$ & $\left\{\mathrm{x}^{\prime}, \mathrm{y}\right\}$
\end{tabular}

Consider the following steps in the algorithm.

Step 0 Define $P_{h}^{0}=P_{h}$.

Step 1 Consider the first violation as $\left(y^{\prime}, x,\left\{x^{\prime}\right\}\right)$ and consider all the contracts $\tilde{Y}$ such that $\mathrm{d}(x)$ is chosen by the hospital. We have the following list for $\tilde{Y}, C_{h}^{0}(\tilde{Y})$, and $C_{h}^{1}(\tilde{Y})$.

\footnotetext{
${ }^{23}$ This is different from the computation time for running this algorithm. It has exponential time complexity in the number of contracts. Each step in the algorithm above searches through $2^{|X|}-1$ subsets of the set of contracts and alters the preferences to arrive at the preference $P_{h}^{i}$ at the end of the step.
} 


$\begin{array}{ccc}\tilde{Y} & C_{h}^{0}(\tilde{Y}) & C_{h}^{1}(\tilde{Y}) \\ \{x\} & \{x\} & \{x\} \\ \left\{x^{\prime}\right\} & \left\{x^{\prime}\right\} & \left\{x^{\prime}\right\} \\ \left\{x, x^{\prime}\right\} & \left\{x^{\prime}\right\} & \left\{x, x^{\prime}\right\} \\ \{x, y\} & \{x, y\} & \{x, y\} \\ \left\{x, y^{\prime}\right\} & \left\{x, y^{\prime}\right\} & \left\{x, y^{\prime}\right\} \\ \left\{x^{\prime}, y\right\} & \left\{x^{\prime}, y\right\} & \left\{x^{\prime}, y\right\} \\ \left\{x^{\prime}, y^{\prime}\right\} & \left\{x^{\prime}, y^{\prime}\right\} & \left\{x^{\prime}, y^{\prime}\right\} \\ \left\{x, y, y^{\prime}\right\} & \left\{x, y^{\prime}\right\} & \left\{x, y^{\prime}\right\} \\ \left\{x^{\prime}, y, y^{\prime}\right\} & \left\{x^{\prime}, y\right\} & \left\{x^{\prime}, y\right\} \\ \left\{x, x^{\prime}, y\right\} & \left\{x^{\prime}, y\right\} & \left\{x, x^{\prime}, y\right\} \\ \left\{x, x^{\prime}, y^{\prime}\right\} & \left\{x, y^{\prime}\right\} & \left\{x, x^{\prime}, y^{\prime}\right\} \\ \left\{x, x^{\prime}, y, y^{\prime}\right\} & \left\{x, y^{\prime}\right\} & \left\{x, x^{\prime}, y^{\prime}\right\}\end{array}$

Although the list above is long, it materially impacts at only three places and we obtain $P_{h}^{1}$ as follows.

$$
\begin{aligned}
P_{h}^{1}: & \left\{x, x^{\prime}, y^{\prime}\right\} \succ\left\{x, y^{\prime}\right\} \succ\left\{x, x^{\prime}, y\right\} \succ\left\{x^{\prime}, y\right\} \succ\left\{x^{\prime}, y^{\prime}\right\} \\
& \succ\{x, y\} \succ\left\{x, x^{\prime}\right\} \succ\left\{x^{\prime}\right\} \succ\left\{y^{\prime}\right\} \succ\{x\} \succ\{y\}
\end{aligned}
$$

Step 2 The second violation was also corrected through the first step. Now consider the third violation $\left(x^{\prime}, y,\left\{y^{\prime}\right\}\right)$ and consider all the contracts $\tilde{Y}$ such that $\mathrm{d}(y)$ is chosen by the hospital. We have the following list for $\tilde{Y}, C_{h}^{0}(\tilde{Y})$, and $C_{h}^{1}(\tilde{Y})$.

\begin{tabular}{ccc}
$\tilde{Y}$ & $C_{h}^{1}(\tilde{Y})$ & $C_{h}^{2}(\tilde{Y})$ \\
\hline$\{y\}$ & $\{y\}$ & $\{y\}$ \\
$\left\{y^{\prime}\right\}$ & $\left\{y^{\prime}\right\}$ & $\left\{y^{\prime}\right\}$ \\
$\left\{y, y^{\prime}\right\}$ & $\left\{y^{\prime}\right\}$ & $\left\{y, y^{\prime}\right\}$ \\
$\{x, y\}$ & $\{x, y\}$ & $\{x, y\}$ \\
$\left\{x, y^{\prime}\right\}$ & $\left\{x, y^{\prime}\right\}$ & $\left\{x, y^{\prime}\right\}$ \\
$\left\{x^{\prime}, y\right\}$ & $\left\{x^{\prime}, y\right\}$ & $\left\{x^{\prime}, y\right\}$ \\
$\left\{x^{\prime}, y^{\prime}\right\}$ & $\left\{x^{\prime}, y^{\prime}\right\}$ & $\left\{x^{\prime}, y^{\prime}\right\}$ \\
$\left\{x, y, y^{\prime}\right\}$ & $\left\{x, y^{\prime}\right\}$ & $\left\{x, y, y^{\prime}\right\}$ \\
$\left\{x^{\prime}, y, y^{\prime}\right\}$ & $\left\{x^{\prime}, y\right\}$ & $\left\{x^{\prime}, y, y^{\prime}\right\}$ \\
$\left\{x, x^{\prime}, y\right\}$ & $\left\{x, x^{\prime}, y\right\}$ & $\left\{x, x^{\prime}, y\right\}$ \\
$\left\{x, x^{\prime}, y^{\prime}\right\}$ & $\left\{x, x^{\prime}, y^{\prime}\right\}$ & $\left\{x, x^{\prime}, y^{\prime}\right\}$ \\
$\left\{x, x^{\prime}, y, y^{\prime}\right\}$ & $\left\{x, x^{\prime}, y^{\prime}\right\}$ & $\left\{x, x^{\prime}, y, y^{\prime}\right\}$
\end{tabular}


Although the list above is long, it materially impacts at four places and we obtain $P_{h}^{2}$ as follows.

$$
\begin{aligned}
P_{h}^{2}: & \left\{x, x^{\prime}, y, y^{\prime}\right\} \succ\left\{x, x^{\prime}, y^{\prime}\right\} \succ\left\{x, y, y^{\prime}\right\} \succ\left\{x, y^{\prime}\right\} \succ\left\{x, x^{\prime}, y\right\} \succ\left\{x^{\prime}, y, y^{\prime}\right\} \succ\left\{x^{\prime}, y\right\} \\
& \succ\left\{x^{\prime}, y^{\prime}\right\} \succ\{x, y\} \succ\left\{x, x^{\prime}\right\} \succ\left\{x^{\prime}\right\} \succ\left\{y, y^{\prime}\right\} \succ\left\{y^{\prime}\right\} \succ\{x\} \succ\{y\}
\end{aligned}
$$

Now there are no more violations in $P_{h}^{2}$ and it satisfies the substitutability property and is a completion of preferences $P_{h}$. Hence, $\bar{P}_{h}=P_{h}^{2}$ is a substitutable completion of $P_{h}$.

\section{$5 \quad$ Properties of stable matchings}

The set of stable matchings satisfy certain properties, like the existence of a doctoroptimal stable matching, a lattice structure, etc. under suitable restrictions on the preferences of the hospitals. We can summarize a few key properties in various settings.

\begin{tabular}{|l|l|l|}
\hline Setting & $\begin{array}{l}\text { Preferences } \\
\text { of all agents }\end{array}$ & Properties \\
\hline Many-to-many & Substitutable & $\begin{array}{l}\Rightarrow \text { Existence of doctor-optimal stable matching } \\
\Rightarrow \text { Existence of doctor-pessimal stable matching } \\
\Rightarrow \text { Existence of a Lattice } \\
+ \text { Law of Aggregate Demand } \Rightarrow \text { Rural Hospital Theorem }\end{array}$ \\
\hline Many-to-one & $\begin{array}{l}\text { Substitutably } \\
\text { completable }\end{array}$ & $\begin{array}{l}\Rightarrow \exists \text { a completion in the many-to-many } \\
\text { setting which is substitutable }\end{array}$ \\
\hline Many-to-one & $\begin{array}{l}\text { Unilaterally } \\
\text { Substitutable }\end{array}$ & $\begin{array}{l}\text { झ Existence of doctor-optimal stable matching } \\
\text { \# Existence of a Lattice } \\
\text { + Law of Aggregate Demand } \Rightarrow \text { Rural Hospital Theorem }\end{array}$ \\
\hline
\end{tabular}

We now know that a unilaterally substitutable preference has a related substitutable preference in the many-to-many setting. Moreover, all the stable matchings in the corresponding many-to-many setting with the substitutable completions of preferences are stable under the original many-to-one setting. However, with unilaterally substitutable preferences, the existence of a lattice structure or a doctorpessimal matching is not guaranteed. This might appear puzzling given that these 
results hold in the many-to-many setting when the preferences for all agents had the substitutability property.

The resolution to this puzzle lies in the fact that under substitutably completed (many-to-many) preferences, only a subset of stable matchings under the original (many-to-one) preferences continue to remain stable. ${ }^{24}$

Consider the following example ${ }^{25}$ where the unilateral substitutability condition is satisfied but not the substitutability condition.

$$
\begin{array}{rlrl}
P_{h}: & \left\{x, y^{\prime \prime}\right\} \succ\left\{x^{\prime \prime}, y\right\} \succ\left\{x^{\prime}, y^{\prime}\right\} & P_{d 1} \quad:\left\{x^{\prime}\right\} \succ\{x\} \succ\left\{x^{\prime \prime}\right\} \succ \emptyset_{d 1} \\
& \succ\left\{x^{\prime \prime}, y^{\prime \prime}\right\} \succ\left\{x^{\prime \prime}, y^{\prime}\right\} \succ\left\{x^{\prime}, y^{\prime \prime}\right\} & P_{d 2} & : \quad\left\{y^{\prime}\right\} \succ\{y\} \succ\left\{y^{\prime \prime}\right\} \succ \emptyset_{d 2} \\
& \succ\left\{x^{\prime}, y\right\} \succ\left\{x, y^{\prime}\right\} \succ\{x, y\} & & \\
& \succ\left\{x^{\prime \prime}\right\} \succ\left\{y^{\prime \prime}\right\} \succ\left\{x^{\prime}\right\} \succ\left\{y^{\prime}\right\} & & \\
& \succ\{x\} \succ\{y\} \succ \emptyset & &
\end{array}
$$

Hatfield and Kojima [2010] show that there is a doctor-optimal stable matching $\left\{x^{\prime}, y^{\prime}\right\}$ and two other stable matchings, $\left\{x^{\prime \prime}, y\right\}$ and $\left\{x, y^{\prime \prime}\right\}$, none of which is doctorpessimal. However, under the completed preferences (using the above algorithm) we would have $\left\{x^{\prime}, y^{\prime}\right\}$ as the one and only stable matching. It is the doctor optimal stable matching and also the doctor pessimal stable matching among the set of stable matchings under the completed preference.

\section{Conclusion}

In these closing remarks, we highlight two points that have not been discussed so far. First, on a technical note, there could be more than one completion of preferences which are substitutable, and the algorithm described above arrives at just one of the possible completions. Second, the substitutably completable preferences are puzzling and are different from other known necessary and various sufficient conditions for stability. This is because substitutable completability is defined implicitly and not described in terms of choices made by the hospital. Through this work, we provide a connection with the unilateral substitutability property, which is defined explicitly.

\footnotetext{
${ }^{24}$ The notion of stability used here is the one for many-to-many preferences as defined in Hatfield and Kominers [2013] Definition 2. An allocation $A \subseteq X$ is stable (with respect to $X$ ) if it is (i) Individually Rational for all $f \in D \cup H C_{f}(A)=A_{f}$ and (ii) Unblocked there does not exist a nonempty blocking set $Z \subseteq X$ such that $Z \cap A=\emptyset$ and for all $f \in \mathrm{d}(A) \cup \mathrm{h}(A), A_{f} \subseteq C_{f}(A \cup Z)$. Also, it is under this notion of stability that the set of stable matchings in many-to-many preferences have a lattice structure.

${ }^{25}$ From page 1717 of Hatfield and Kojima [2010]
} 
Hatfield and Kominers [2014] use techniques to arrive at a completion for specific preferences, namely, slot-based preferences and task-based preferences. We provide an algorithm for new sub-domains of the substitutably completable preferences. In doing so, we further clarify the connection between known concepts. A generalized algorithm for reaching a completion of preferences remains elusive as does a general characterization of the substitutably completable preferences. The hidden structure in various conditions which guarantee the existence of stability, which is yet to be uncovered, will close the lacuna in our understanding. We present a small step in this direction and hope that further research will provide us the necessary and sufficient condition for the existence of stable matchings.

\section{Appendix}

Claim 1 The maximal subset of $\tilde{Y}$ with only unrejected contracts with a doctor, say $d_{1}$ is uniquely defined.

Proof Consider two sets $\hat{Y}_{1}$ and $\hat{Y}_{2}$ both being the maximal subsets as defined above in definition 3.1. We also have $\hat{Y}_{1} \neq \hat{Y}_{2}$ and they can not ordered by set inclusion. Define $\hat{Y}=\hat{Y}_{1} \cup \hat{Y}_{2}$. From the definition above we know that $\hat{Y}_{1} \backslash\left[\hat{Y}_{1}\right]_{d_{1}}=$ $\tilde{Y} \backslash \tilde{Y}_{d_{1}}=\hat{Y}_{2} \backslash\left[\hat{Y}_{2}\right]_{d_{1}}=\hat{Y} \backslash \hat{Y}_{d_{1}}$. Moreover $\hat{Y}$ also satisfies the second requirement which would mean that neither $\hat{Y}_{1}$ nor $\hat{Y}_{2}$ are maximal. To prove a contradiction suppose not. There exists a $Y^{\prime \prime} \subseteq \hat{Y}$ such that $d_{1} \in \mathrm{d}\left(Y^{\prime \prime}\right)$ but $d_{1} \notin \mathrm{d}\left[C_{h}\left(Y^{\prime \prime}\right]\right.$. Consider any contract with doctor $d_{1}$ in such a $Y^{\prime \prime}$ and let us call it $z$. It is clear that $z \in \hat{Y}_{1}$ or $z \in \hat{Y}_{2}$. Consider the set $Y_{1}=Y^{\prime \prime} \backslash\left[Y^{\prime \prime}\right]_{d_{1}} \cup\{z\}$. By IRC, we have $d_{1} \notin \mathrm{d}\left[C_{h}\left(Y_{1}\right)\right]$. However, since $Y^{\prime \prime} \backslash\left[Y^{\prime \prime}\right]_{d_{1}} \subseteq \tilde{Y} \backslash \tilde{Y}_{d_{1}}$ we would have $Y_{1} \subseteq \hat{Y}_{1}$ or $Y_{1} \subseteq \hat{Y}_{2}$. This would violate the second requirement for the above definition for either $\hat{Y}_{1}$ or $\hat{Y}_{2}$ and this would contradict our assumption.

Claim 2 There exists a preference relation $P_{h}^{i}$ over the subsets of $X_{h}$ such that $C_{h}^{i}$ defined in step $i$ in the completion algorithm 3.1 corresponds to $P_{h}^{i}$.

Proof We provide a proof by induction. This statement is trivially true by assumption for $i=0$. Assume that it holds for $i-1$ for $i \geq 1$. We prove that it holds for $i$ constructively.

$i .1$ Define $\hat{P} \equiv P_{h}^{i-1}$ and $\hat{C}(\cdot) \equiv C_{h}^{i-1}(\cdot)$ for the hospital $h$ corresponding to the preference relation $P_{h}^{i-1}$. Also define $\hat{X} \equiv 2^{X_{h}} \backslash\{\varnothing\}$.

$i .2$ If $\hat{X}=\varnothing$ then $P_{h}^{i} \equiv \hat{P}$ and Exit this step $i$ in the completion algorithm 3.1 and go to next step $i+1$. Else choose $\tilde{Y} \in \hat{X}$ and go to step $i .3$. 
$i .3$ Redefine $\hat{X} \equiv \hat{X} \backslash\{\tilde{Y}\}$.

$i .4$ If $\mathrm{d}\left(z_{i}\right) \notin \mathrm{d}[\hat{C}(\tilde{Y})]$ then go to step $i .2$. Else go to step $i .5$.

$i .5$ Find the required maximal subset $\hat{Y}$ for $\tilde{Y}$ and define $Y^{\prime \prime}=\hat{C}(\tilde{Y}) \cup \hat{Y}_{\mathrm{d}\left(z_{i}\right)}$ and go to step $i .6$.

$i .6$ If $\hat{C}(\tilde{Y})=Y^{\prime \prime}$ then go to step $i .2$. Else go to step $i .7$.

i.7 Define a preference relation $\hat{\hat{P}}$ by moving $Y^{\prime \prime}$ so that it is (just) better than $\hat{C}(\tilde{Y})$, i.e. $\forall \tilde{Y} \neq Y^{\prime \prime}$ and $\subseteq X_{h}$ we should have the following.

$$
\tilde{\tilde{Y}} \succ Y^{\prime \prime} \text { under } \hat{\hat{P}} \Longleftrightarrow \tilde{\tilde{Y}} \succ \hat{C}(\tilde{Y}) \text { under } \hat{P}
$$

Clearly $\hat{\hat{C}}(\tilde{Y})=Y^{\prime \prime}$ under $\hat{\hat{P}}$. Go to next step $i .8$.

$i .8$ Define $\hat{P} \equiv \hat{\hat{P}}$ and $\hat{C}(\cdot)$ corresponds to the new preference relation $\hat{P}$. Go to step $i .2$

The above construction takes each non-empty subset of $X_{h}$ one at a time and modifies the preference relationship iteratively.

In a given step $i$ where the preferences are being modified to fix the substitutability violation concerning the doctor $\mathrm{d}\left(z_{i}\right)$ the following statements follow immediately from the algorithm:

- Preference is modified only for sets where a $\mathrm{d}\left(z_{i}\right)$ contract is in the chosen set.

- When preference is modified for such sets $\tilde{Y}$ all $\mathrm{d}\left(z_{i}\right)$ contracts in $\hat{Y}$ are added to the new chosen set by moving this set as being better than the old chosen set.

- Once the set is moved in the preference relation to be better than the old chosen set then in the subsequent iterations of the algorithm above it continues to be better than the old chosen set, which in turn was better than all the feasible and infeasible subsets of the set, in a given step.

Thus at the end of the algorithm above, we have a preference relation $P_{h}^{i}$ and a choice function $C_{h}^{i}(\cdot)$ which agrees with the description in step $i$ of the completion algorithm.

Proof of Proposition 3.1 We first prove that at step $i$, with a substitutability violation $\left(x_{i}, z_{i}, Y_{i}\right)$, there exist no substitutability violation $\left(\tilde{x}_{i}, \tilde{z}_{i}, \tilde{Y}_{i}\right)$ under $P_{h}^{i}$ such 
that $\mathrm{d}\left(\tilde{z}_{i}\right)=\mathrm{d}\left(z_{i}\right)$. Consider such a substitutability violation involving the doctor of the recalled contract under $P_{h}^{i-1}$. Using the same technique as in proof of Lemma 3.2, using part $I$ we can claim that $\exists \hat{z}_{i} \in C_{h}^{i-1}\left(\tilde{Y}_{i} \cup\left\{\tilde{z}_{i}\right\}\right)$ such that $\mathrm{d}\left(\hat{z}_{i}\right)=\mathrm{d}\left(\tilde{z}_{i}\right)=\mathrm{d}\left(z_{i}\right)$. Proceeding along the same lines of part $I I$, we would establish that for the maximal subset $\tilde{Y}_{i}^{\prime}$ of $\tilde{Y}_{i}, \tilde{z}_{i} \in \tilde{Y}_{i}^{\prime}$ and hence is added in step $i$. The substitutability violation no longer exists after step $i$. Thus $\left(\tilde{x}_{i}, \tilde{z}_{i}, \tilde{Y}_{i}\right)$ is not a substitutability violation under $P_{h}^{i}$. Thus at each step all the violations involving a given doctor are fixed. By Lemma 3.1, we know that no new violations are created and hence there will be no new violations involving the doctor $\mathrm{d}\left(z_{i}\right)$ in the subsequent steps. This proves that the algorithm above identifies up to $|D|$ violations and completes in $|D|$ steps.

\section{References}

Orhan Aygün and Tayfun Sönmez. Matching with contracts: Comment. American Economic Review, 103:2050-2051, 2013a.

Orhan Aygün and Tayfun Sönmez. Matching with contracts: Critical role of irrelevance of rejected contracts. mimeo, Boston College, 2013b. URL https: //www2. bc . edu/ sonmezt/AygunSonmez2012a.pdf.

Vincent P. Crawford and Elsie Marie Knoer. Job matching with heterogeneous firms and workers. Econometrica, 49(2):pp. 437-450, 1981. ISSN 00129682. URL http://www. jstor.org/stable/1913320.

Federico Echenique. Contracts versus salaries in matching. American Economic Review, 102(1):594-601, 2012. doi: 10.1257/aer.102.1.594. URL http://www . aeaweb.org/articles.php?doi=10.1257/aer.102.1.594.

David Gale and Lloyd Shapley. College admissions and the stability of marriage. American Mathematical Monthly, 69:9-15, 1962.

John W. Hatfield and Fuhito Kojima. Matching with contracts: Comment. American Economic Review, 98:1189-1194, 2008.

John W. Hatfield and Fuhito Kojima. Substitutes and stability for matching with contracts. Journal of Economic Theory, 145(5):1704-1723, 2010.

John W. Hatfield and Scott D. Kominers. Matching in networks with bilateral contracts. American Economic Journal: Microeconomics, 4(1):176-208, 2012. 
John W. Hatfield and Scott D. Kominers. Contract design and stability in many-tomany matching. Harvard Business School Working Paper, 2013.

John W. Hatfield and Scott D. Kominers. Hidden substitutes. Mimeo, Harvard University, August 2014.

John W. Hatfield and Paul Milgrom. Matching with contracts. American Economic Review, 95:913-935, 2005.

John William Hatfield, Nicole Immorlica, and Scott Duke Kominers. Testing substitutability. Games and Economic Behavior, 75(2):639 - 645, 2012. ISSN 0899-8256. doi: http://dx.doi.org/10.1016/j.geb.2011.11.007. URL http://www . sciencedirect.com/science/article/pii/S0899825611001850.

Yuichiro Kamada and Fuhito Kojima. Stability and strategy-proofness for matching with constraints: A problem in the japanese medical match and its solution. The American Economic Review, 102(3):pp. 366-370, 2012. ISSN 00028282. URL http: //www . jstor .org/stable/23245558.

Yuichiro Kamada and Fuhito Kojima. Efficient matching under distributional constraints: Theory and applications. American Economic Review, 105(1):67-99, 2015a. doi: 10.1257/aer.20101552. URL http://www.aeaweb.org/articles . php?doi=10.1257/aer . 20101552.

Yuichiro Kamada and Fuhito Kojima. General theory of matching under distributional constraints. Mimeo, Haas School of Business and Stanford University, January 2015b.

Alexander S. Kelso and Vincent P. Crawford. Job matching, coalition formation, and gross substitutes. Econometrica, 50(6):pp. 1483-1504, 1982. ISSN 00129682. URL http: //www . jstor .org/stable/1913392.

Scott D. Kominers and Tayfun Sönmez. Designing for diversity in matching. mimeo, Harvard University and Boston College, 2013. URL http://www.scottkom.com/ articles/Kominers_Sonmez_Designing_for_Diversity_in_Matching.pdf.

Michael Ostrovsky. Stability in supply chain networks. American Economic Review, 98:897-923, 2008.

Alvin E. Roth. Stability and polarization of interests in job matching. Econometrica, 52(1):pp. 47-58, 1984. ISSN 00129682. URL http://www.jstor.org/stable/ 1911460. 
Alvin E. Roth. New physicians: A natural experiment in market organization. Science, 250(4987):pp. 1524-1528, 1990. ISSN 00368075. URL http://www. jstor. org/stable/2878330.

Tayfun Sönmez. Bidding for army career specialities: Improving the ROTC branching mechanism. Journal of Political Economy, 121(1):186-219, 2013.

Tayfun Sönmez and Tobias Switzer. Matching with (branch-of-choice) contracts at the united states military academy. Econometrica, 81(2):451-488, 2013. 